\title{
Fufang-Zhenzhu-Tiaozhi Capsule reduces restenosis via the downregulation of NF- kappaB and inflammatory factors in rabbits
}

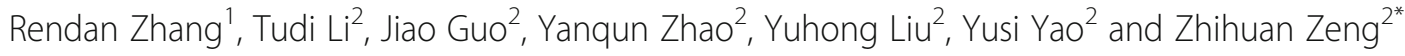

\begin{abstract}
Background: To investigate the effects of a Chinese herbal medicine Fufang-Zhenzhu Tiaozhi Capsule (FTZ) on restenosis and elucidate the mechanism of action.

Methods: A restenosis model was established by balloon rubbing the endothelium of the abdominal aorta followed by high fat diet. Rabbits were divided into blank control group, restenosis group, FTZ group $(0.66 \mathrm{mg} / \mathrm{kg} / \mathrm{day})$, atorvastatin group ( $5 \mathrm{mg} / \mathrm{kg} /$ day $)$ and FTZ + atorvastatin group $(n=8)$. Vascular stenosis was analyzed by $X$-ray. Serum levels of chemokines and cytokines interleukin-1 (IL-1), interleukin-6 (IL-6), interleukin-8 (IL-8), interleukin-12 (IL-12), C-reactive protein (CRP), tumor necrosis factor-alpha (TNF-a), monocyte chemoattractant protein-1 (MCP-1), and intercellular adhesion molecule-1 (ICAM-1) were measured by ELISA. The levels of NF-KB, IKB-a, P-IKBa, IKK- $\mathrm{a}$, and $\mathrm{P}-\mathrm{IKK} / \mathrm{\beta}$ from injured abdominal arteries were detected by Western blotting.
\end{abstract}

Results: Restenosis was induced successfully via abdominal artery balloon injuries and high fat diet. Restenosis was significantly decreased in FTZ group compared with restenosis group $(P<0.05)$. FTZ group had markedly reduced serum lipid levels $(P<0.05)$. In addition, the levels of TNF-a, IL-1, IL-6, IL-8, IL-12, ICAM-1 and MCP-1 decreased by FTZ treatment $(P<0.05)$. The expression of NF-kB in the atherosclerotic lesions was significantly attenuated in FTZ group $(P<0.05)$.

Conclusion: FTZ could reduce restenosis via reducing NF-KB activity and inflammatory factor expression within the atherosclerotic lesion in a rabbit restenosis model. FTZ may be a new therapeutic agent for restenosis.

Keywords: FTZ, NF-kB, Animal model, Restenosis, Inflammation

\section{Introduction}

Percutaneous coronary intervention (PCI) has been widely used in the treatment of coronary artery disease. Despite the decrease of restenosis in the era of stenting, post-operative arterial restenosis remains a problem [1]. Restenosis is characterized by intimal hyperplasia, smooth muscle cell proliferation, and vascular renarrowing, and the rate of restenosis following PCI is higher in patients with hyperlipidemia, and the recurrence time of angina is highly related to the history of hyperlipidemia [2-4]. Recent studies have demonstrated that obese adipose tissue accelerates the progression of inflammation and promotes

\footnotetext{
*Correspondence: gzzh@163.com

${ }^{2}$ Department of Cardiology, The First Affiliated Hospital of Guangdong

Pharmaceutical University, Guangzhou 510080, Guangdong, China

Full list of author information is available at the end of the article
}

the development of atherosclerosis [5]. Lipid lowering therapy, especially targeting low-density lipoprotein cholesterol (LDL-C) $<70 \mathrm{mg} / \mathrm{dL}$, can alter the pattern of restenosis after PCI [6].

According to current guidelines, lipid lowering agents including statins should be prescribed in subjects with coronary artery disease after PCI [7]. However, these agents have side effects. Fufang-Zhenzhu Tiaozhi Capsule (FTZ) is a Chinese herbal medicine prescription including Rhizoma Coptidis, Radix Salvia Miltiorrhiza, Radix Notoginseng, Fructus Ligustri Lucidi, Herba Cirsii Jeponici, Cortex Eucommiae, Fructus Citri Sarcodactylis and Radix Atractylodes Macrocephala, and has been used clinically to reduce serum levels of total cholesterol (TC), triglycerides (TG) and LDL-C, while increase high-density lipoprotein cholesterol (HDL-C) in subjects

(C) The Author(s). 2018 Open Access This article is distributed under the terms of the Creative Commons Attribution 4.0 International License (http://creativecommons.org/licenses/by/4.0/), which permits unrestricted use, distribution, and 
with dyslipidemia [8]. FTZ exhibits a variety of pharmacological properties, such as alleviating inflammation, regulating blood coagulation, improving endothelial function, and inhibiting the formation and development of atherosclerosis plaque [9]. However, whether FTZ has the efficacy to reduce restenosis and the underlying mechanisms are unknown. The aim of this study was to investigate the effects of FTZ on restenosis and explore whether it is mediated by the regulation of inflammation.

\section{Materials and methods}

\section{Drugs}

Herbs in FTZ [composed of Ligustri Lucidi Fructus (LLF), Salviae Miltiorrhizae Radix et Rhizoma (SMR), Coptidis Rhizoma (CR),Notoginseng Radix et Rhizoma (NRR), Atractylodis Macrocephalae Rhizoma (AMR), Cirsii Japonici Herba Radix (CJHR), Citri Sarcodactylis Fructus (CSF) and Eucommiae Cortex (EC)] were provided by Zhixin Chinese Herbal Medicine Co., Ltd. (Guangzhou, China) [10]. FTZ was gifted by GUANGDONG METABOLIC DISEASES RESEARCH CENTER OF INTEGRATED CHINESE AND WESTERN MEDICINE (Guangzhou, China). The quality analysis of FTZ extract was performed by HPLC fingerprint method as previously reported [11]. A voucher specimen was deposited in the Institute of Chinese Medicine of Guangdong Pharmaceutical University. Atorvastatin as the control drug was provided by Pfizer (USA).

\section{Animals}

Forty male New Zealand rabbits $(1.8-2.2 \mathrm{~kg})$ were supplied by Guangdong Medicinal Laboratory Animal Center, Guangzhou, China (Certification: SYXK Guangdong 2012-0125). All animal procedures were performed in accordance with the protocols approved by the Committee for Supervision of Animal Experiments of Guangdong Pharmaceutical University (Guangzhou, China). After one week of acclimatization, forty rabbits were randomized into five groups $(n=8)$ : blank group, restenosis group, FTZ group, atorvastatin group and FTZ + atorvastatin group.

Except for blank group receiving shame operation, the other four groups were given the following operations: the animals were anesthetized with auricular vein injection of pentobarbital sodium $(1 \mathrm{mg} / \mathrm{kg})$ and supine position fixation. Then $6 \mathrm{~F}(1 \mathrm{~F}=0.33 \mathrm{~mm})$ artery sheath tubes (Terumo Corporation, Japan) were introduced by guide wires through the femoral arteries and advanced into the abdominal arteries under the digital subtraction X-ray machine (Artis zee III ceiling, Siemens, Germany). The Sprinter $3.0 \mathrm{~mm} \times 20 \mathrm{~mm}$ balloons (Metronic, USA) were inflated with $303.975 \mathrm{kPa}$ and then moved up and down through the abdominal aortas three times to ensure adequate damage of the endothelium. The wounds were adequately pressurized and enswathed. Penicillin sodium was injected inside the thighs for 3 days to avoid infection.
Then, the rabbits were fed high fat diet $(1.5 \%$ cholesterol, $0.5 \%$ sodium cholate, $8 \%$ lard, $10 \%$ egg yolk powder) for 4 weeks to form atherosclerosis. In order to prevent diarrheal, the rabbits were fed yeast tablets $0.2 \mathrm{~g} /$ day.

After 4 weeks of high fat diet, the four groups underwent angiography and rubbing of endothelium by balloon to generate restenosis. Then, the rabbits were fed regular chow for another 4 weeks and given corresponding drugs including FTZ $(0.66 \mathrm{mg} / \mathrm{kg} /$ day $)$ in FTZ group, atorvastatin $(5 \mathrm{mg} / \mathrm{kg} /$ day $)$ in atorvastatin group $(5 \mathrm{mg} / \mathrm{kg} /$ day $)$, and FTZ $(0.66 \mathrm{mg} / \mathrm{kg} /$ day $)$ plus atorvastatin $(5 \mathrm{mg} / \mathrm{kg} /$ day $)$ in FTZ + atorvastatin group. At the eighth week, angiographic analyses were performed to verify the effects of drug treatment. Then all animals were sacrificed and abdominal aortas were taken for further pathological analysis. Body weights were measured and food intake was monitored weekly. Meanwhile, $10 \mathrm{ml}$ of arterial blood was taken for biochemical analysis.

\section{Angiographic analysis of arterial stenosis}

The imaging results of each group were analyzed by two-dimensional QCA original workstation from digital subtraction X-ray machine (Artis zee III ceiling, Siemens, Germany). Main measurement indicators: (1) the length of two different angles of the target vessel (take the relative more exposure of vascular lesions); (2) Reference Vascular Diameter: The sum of the proximal diameter and distal diameter of the lesion; (3) \% MLD $=(1$-actual diameter $/$ reference diameter $) \times 100 \%$ (The average sum of the diameter stenosis rates of two different angles); (4) \% MLA = (1 - actual stenosis area/reference area) $\times 100 \%$ (The average sum of the area narrow rates of two different angles).

\section{Biochemical analysis}

Blood samples were collected from the abdominal aortas and left at room temperature for coagulation. The serum samples were obtained by centrifugation at $3000 \mathrm{rpm}$ for $15 \mathrm{~min}$ at $4{ }^{\circ} \mathrm{C}$, the separated serum was frozen at $-80^{\circ} \mathrm{C}$ for further analysis. Serum levels of LDL-C, HDL-C, TC, TG, very low-density lipoprotein cholesterol (VLDL-C) and C-reactive protein (CRP) were analyzed by a blood chemistry analyzer (Hitachi 7108ISE, Japan).

\section{Elisa}

The concentrations of TNF-a, IL-1, IL-6, IL-8, IL-12, ICAM-1 and MCP-1 in serum were measured using commercial ELISA kits (Wuhan Huamei Biological Engineering Co., Ltd., China) according to the manufacturer's instructions. The optical density of samples was detected using an automated Spectra Max190 microplate reader (Thermo Fisher, USA) at $450 \mathrm{~nm}$. The levels of cytokines were calculated based on a corresponding standard curve. 


\section{Histomorphometric analysis}

Abdominal aortic tissues of rabbits were harvested for histopathological examination. The tissue samples were washed with saline and fixed in $4 \%$ paraformaldehyde for 48-72 $\mathrm{h}$, dehydrated with graded alcohol, embedded in paraffin and then artery sections $(5 \mu \mathrm{m})$ were stained with hematoxylin-eosin (HE) and immunohistochemical staining. Morphological changes in the aortas of HE were observed with an optical microscope (Olympus, Japan). The intima (IT), the intima-to-media ratios (IMRS) and medial thickness were calculated using the Image ProPlus 6.0 Analyzer software (Media Cybernetics, Silver Spring,

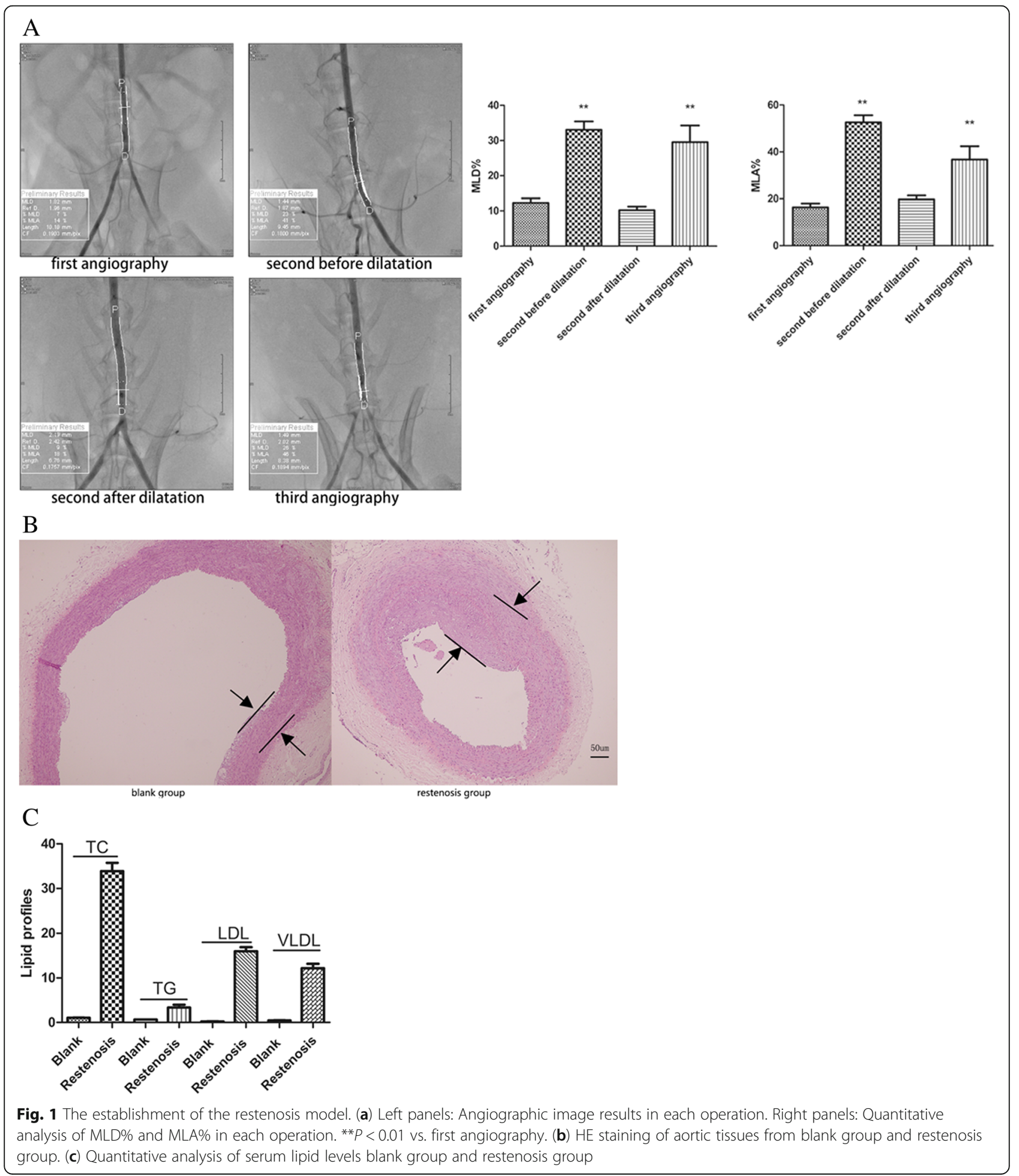


MD, USA). IMRS was used to reflect the intimal hyperplasia of the injured vessels.

\section{Western blot analysis}

Proteins were extracted from injured abdominal arteries and equivalent amounts of protein from each sample were electrophoresed on a 5\% discontinuous polyacrylamide minigel, and transferred onto nitrocellulose membranes. The membranes were blocked at $4{ }^{\circ} \mathrm{C}$ overnight with $5 \%$ non-fat dry milk, then incubated with antibodies for NF- $\mathrm{kB}$ (Thermo Fisher, USA), IкB- $\alpha$, P-IкB $\alpha$, IKK- $\alpha$, and P-IKK $\alpha / \beta$ (all from BIOSS, China) at $4{ }^{\circ} \mathrm{C}$ overnight. The membranes were washed and incubated with secondary antibodies for $30 \mathrm{~min}$ at room temperature. The immune complexes were visualized using the enhanced chemiluminescence method. Protein levels were quantified by using PhotoShop (version 18.0, Adobe Corporation, USA).

\section{Statistical analysis}

Statistical analyses were conducted using SPSS21.0 software. Results were reported as the mean \pm standard deviation and analyzed by analysis of variance (ANOVA) with Tukey's post hoc test. Differences were considered significant at $P<0.05$.

\section{Results}

Successful establishment of restenosis model

As shown in Fig. 1a, MLD\% and MLA\% significantly increased (MLD\%:12.2 $\pm 9.9 \%$ vs. $33.0 \pm 15.6 \%, P<0.01$; MLA\%:16.4 $\pm 11.2 \%$ vs. $52.5 \pm 20.0 \%, P<0.01)$ in second angiography, compared with the first abdominal aortic angiography. MLD\% and MLA\% in the restenosis group were significantly increased in the third operation, compared with the second angiographic after balloon dilatation (MLD\%:29.5 $\pm 11.6 \%$ vs. $10.2 \pm 5.7 \%, P<0.01$; MLA\%:36.7 $\pm 18.7 \%$ vs. $19.7 \pm 9.7 \%, P<0.01$ ).

Furthermore, pathological analysis indicated that there was no thickening and lipid deposition, monolayer cells were close to the inner elastic plate, and the middle vascular smooth muscle cells (SMCs) were arranged neatly in the blank group. However, the restenosis group showed severe neointimal hyperplasia and internal elastic plate discontinuity. The intima was thickened, a large number of SMCs were arranged in a disorderly manner, and foam cells were accumulated in the intima (Fig. 1b). Compared with normal diet rabbits, serum TC, TG, LDL-C, and VLDL-C levels were significantly elevated in high fat diet-fed rabbits (Fig. 1c). Taken together, these data indicate successful establishment of model of atherosclerosis and restenosis.

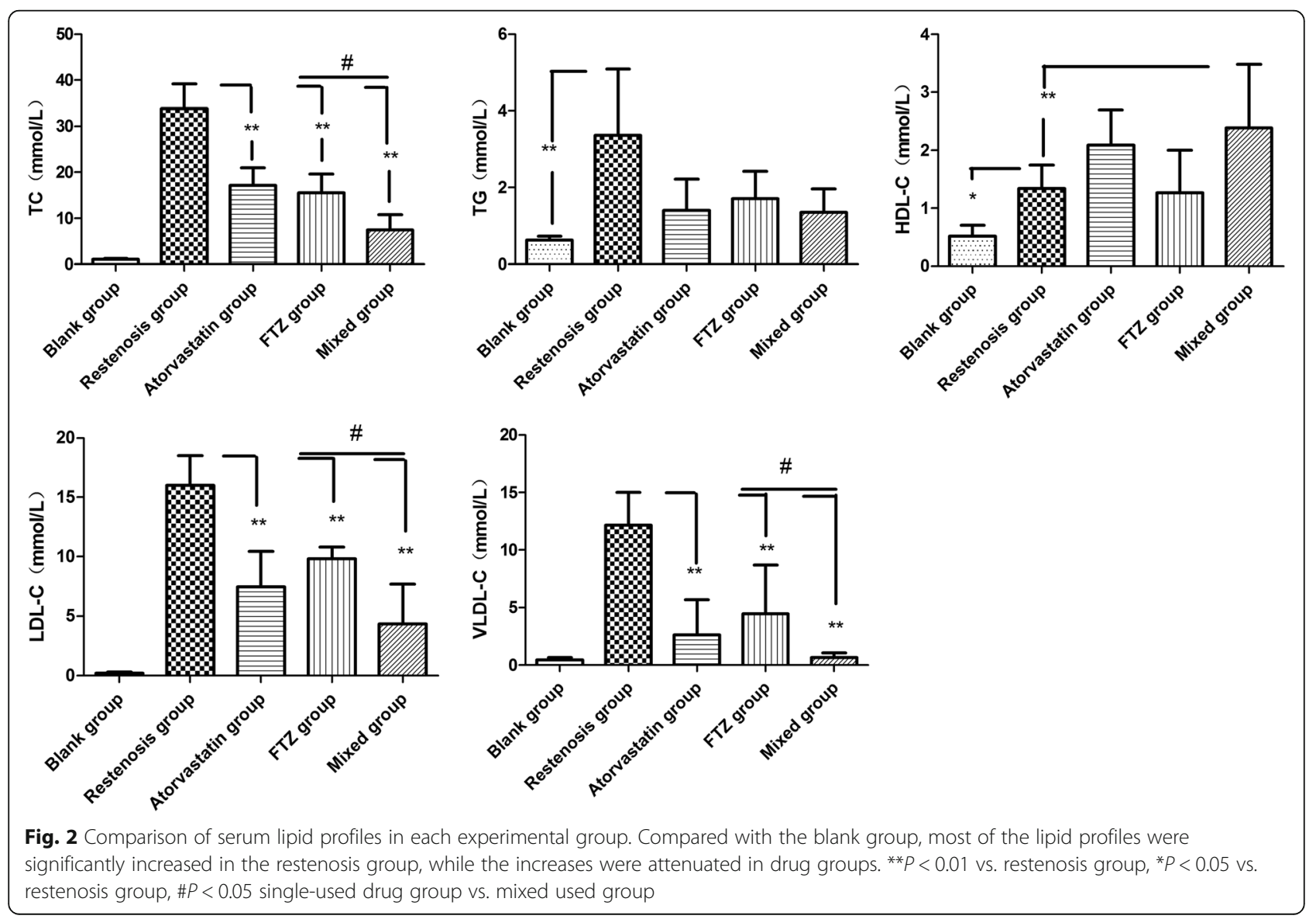




\section{FTZ reduces serum lipid levels of rabbits}

Compared with restenosis group, FTZ and atorvastatin led to significant reduction of serum TC, LDL-C and VLDL-C levels (all $P<0.01$ ) (Fig. 2). In addition, reduced levels of HDL-C in the high fat-fed rabbits were reversed by FTZ and atorvastatin. There were no differences in TC, TG, LDL-C and VLDL-C between FTZ and atorvastatin groups, indicating an equivalent effect of FTZ on lipid profiles compared with atorvastatin. Furthermore, compared with FTZ and atorvastatin group, the levels of TC, LDL-C and
VLDL-C were significantly lower in mixed group $(P<0.05)$, and HDL-C was increased, indicating an improved lipid profiles in combination therapy.

\section{FTZ effectively inhibits vascular restenosis}

Compared with the blank group, MLD\% and MLA\% were significantly increased in the restenosis group (MLD\%:2.2 $\pm 1.2 \%$ vs. $34 \pm 7.2 \%, P<0.01$; MLA\%:4.6 \pm $2.6 \%$ vs. $55.7 \pm 9.0 \%, P<0.01)$. Compared with restenosis

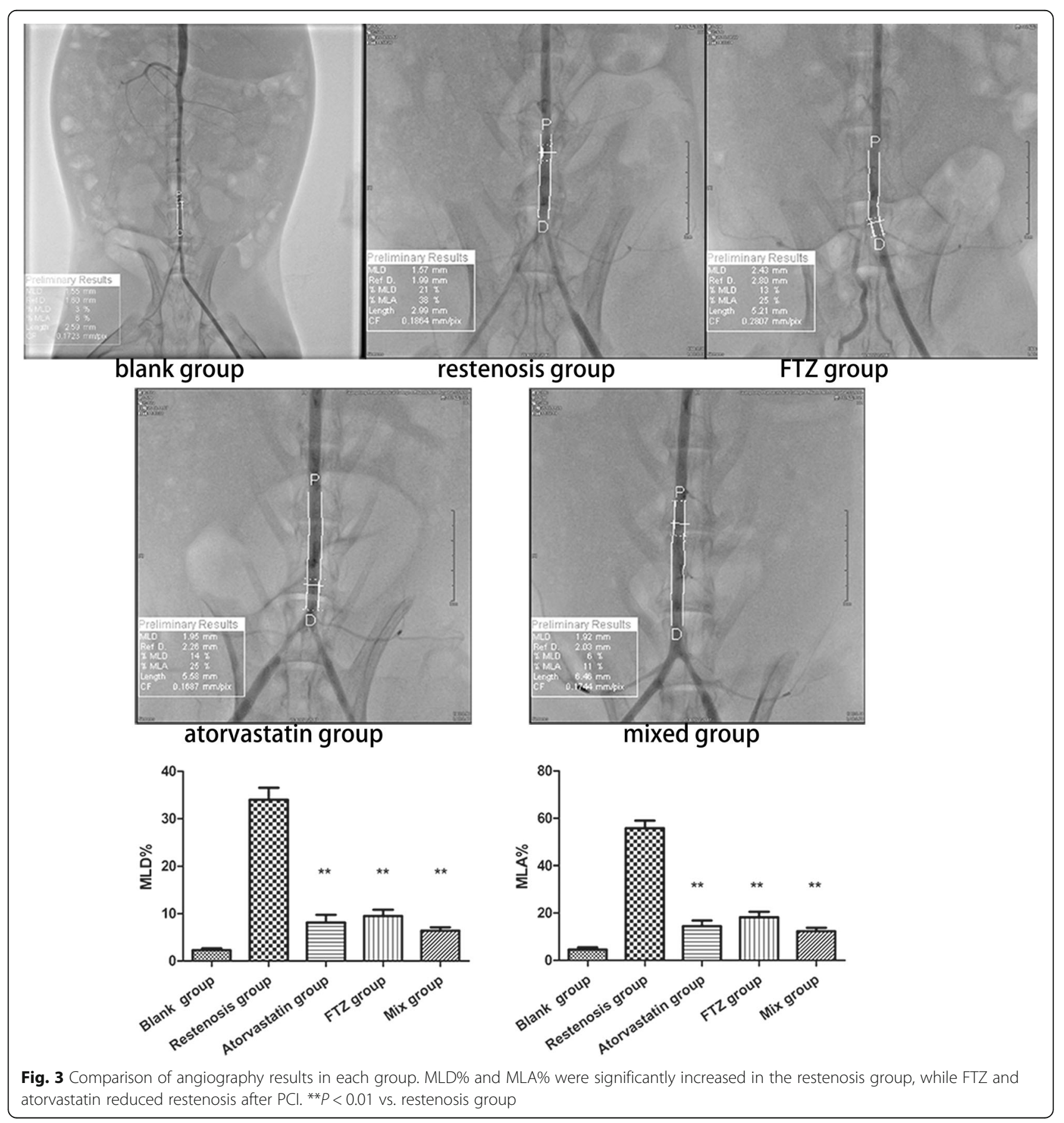


group, MLD\% and MLA\% in FTZ, atorvastatin and mixed groups were significantly decreased (all $P<0.01$ ) (Fig. 3).

As shown in Fig. 4, histopathological assessment showed that IT and IMRS in the restenosis group was significantly thicker than that in the blank group (all $P<0.01)$. Compared with the restenosis group, IT and IMRS of drug groups were significantly decreased (all $P<0.01$ ). The combination of two drugs were better in decreasing IT and IMRS.

\section{FTZ reduces serum cytokines levels}

Compared with the blank group, serum levels of IL-1, IL-6, IL-8, IL-12, CRP, TNF- $\alpha$ and MCP-1 in restenosis group significantly increased $(P<0.01)$, and serum ICAM-1 level significantly increased too $(P<0.05)$ (Table 1$)$. These data indicate a significant inflammatory response in the restenosis group. However, the levels of pro-inflammatory cytokines IL- 1 and IL- 8 and CRP TNF- $\alpha$, MCP- 1 , and ICAM-1 decreased significantly in treatment groups compared with the restenosis group (all $P<0.01$ ). Furthermore, there were no significant differences in the levels of these factors between FTZ and atorvastatin groups, indicating a similar effect in inhibiting inflammatory response. Compared with the single-treatment group, the levels of IL-1 and ICAM-1 were significantly lower in mixed group $(P<0.05)$, which suggested that the combination of two drugs is more effective in inhibiting inflammatory response.

\section{FTZ inhibits NF-KB activation in the aorta}

To understand the mechanism underlying anti-inflammation effects of FTZ, we detected the activation of NF-kB. Western blot analysis showed that the expression of NF- $\kappa B, I \kappa B-\alpha, I K K \alpha$ and P-IKK $\alpha / \beta$ in abdominal aortas of high fat diet-fed rabbits elevated compared to normal diet-fed rabbits, and administration of FTZ markedly reduced the levels of NF-кB, IкB- $\alpha$, IKK $\alpha$, and P-IKK $\alpha / \beta$ (Fig. 5). Atorvastatin group showed similar effects to inhibit the activation of NF- $\mathrm{KB}$. These results suggest that FTZ plays an anti-inflammatory role by reducing the expression of NF- $\mathrm{kB}$ in abdominal aortas.

\section{Discussion}

In this study, a simple, safe, and reproducible model of injury-induced vascular restenosis was established through percutaneous artery puncture. Using this model, we found that FTZ treatment significantly improved vascular restenosis with anti-inflammatory effects in the abdominal arteries.

Hyperlipidemia plays an important role in restenosis. Previous studies have shown that hyperlipidemia

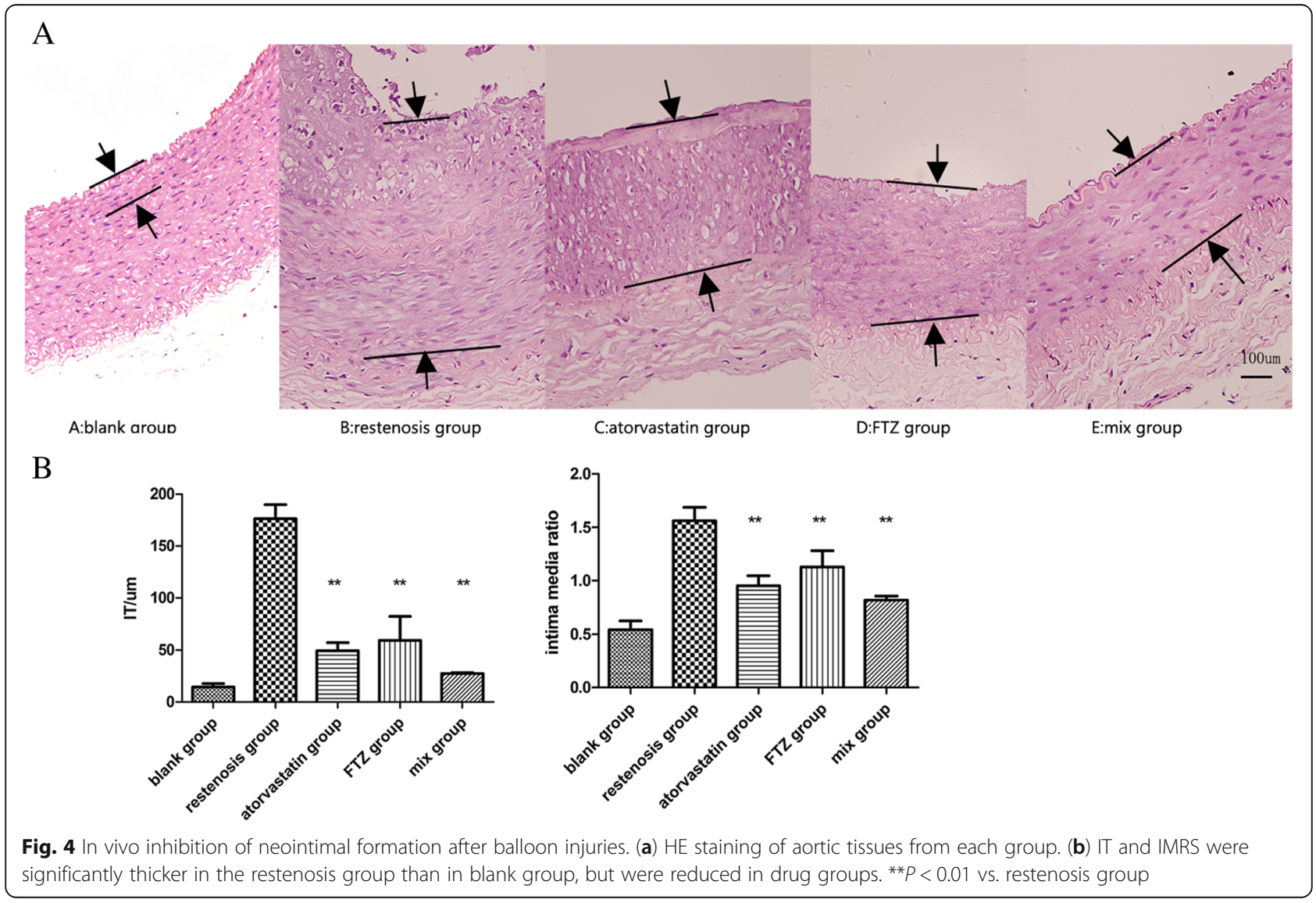


Table 1 Serum levels of inflammatory factors in each group

\begin{tabular}{llllll}
\hline Variable & blank group & restenosis group & atorvastatin group & FTZ group & mixed group \\
\hline IL-1 $(\mathrm{ng} / \mathrm{ml})$ & $122.76 \pm 11.39$ & $161.96 \pm 21.56$ & $94.17 \pm 24.53^{* *}$ & $94.42 \pm 17.77^{* *}$ & $75.13 \pm 14.01^{* * \#}$ \\
IL-6 $(\mathrm{ng} / \mathrm{ml})$ & $339.72 \pm 7.91^{* *}$ & $419.89 \pm 43.35$ & $349.64 \pm 20.1$ & $358.89 \pm 20.92$ & $324.44 \pm 33.61$ \\
IL-8(ng/ml) & $404.26 \pm 109.85$ & $994.9 \pm 165.16$ & $680.71 \pm 53.1^{* *}$ & $605.46 \pm 16.31^{* *}$ & $583.79 \pm 154.89^{* *}$ \\
IL-12(ng/ml) & $175.89 \pm 11.96^{* *}$ & $242.41 \pm 33.32$ & $192.57 \pm 16.45$ & $194.07 \pm 18.76$ & $188.72 \pm 10.45$ \\
CRP $(\mathrm{mg} / \mathrm{L})$ & $0.1 \pm 0.0 x$ & $0.46 \pm 0.05$ & $0.13 \pm 0.04^{* *}$ & $0.16 \pm 0.74^{* *}$ & $0.13 \pm 0.02^{* *}$ \\
MCP-1 $(\mathrm{ng} / \mathrm{ml})$ & $214.15 \pm 42.67$ & $402.88 \pm 41.9$ & $265.92 \pm 43.4^{* *}$ & $265.48 \pm 47.6^{* *}$ & $257.99 \pm 40.99^{* *}$ \\
ICAM-1 $(\mathrm{ng} / \mathrm{ml})$ & $121.93 \pm 8.16$ & $156.57 \pm 20.81$ & $73.37 \pm 44.52^{* *}$ & $49.99 \pm 39.72^{* *}$ & $20.31 \pm 8.93^{* * \#}$ \\
TNF-a $(\mathrm{ng} / \mathrm{ml})$ & $12.69 \pm 3.85$ & $37.67 \pm 20.14$ & $22 \pm 3.95^{* *}$ & $19.45 \pm 2.94^{* *}$ & $14.18 \pm 3.57^{* *}$ \\
\hline
\end{tabular}

${ }^{*} P<0.01$ vs. restenosis group, ${ }^{*} P<0.05$ vs. restenosis group, $\# P<0.05$ means single-used drug group vs. mixed group

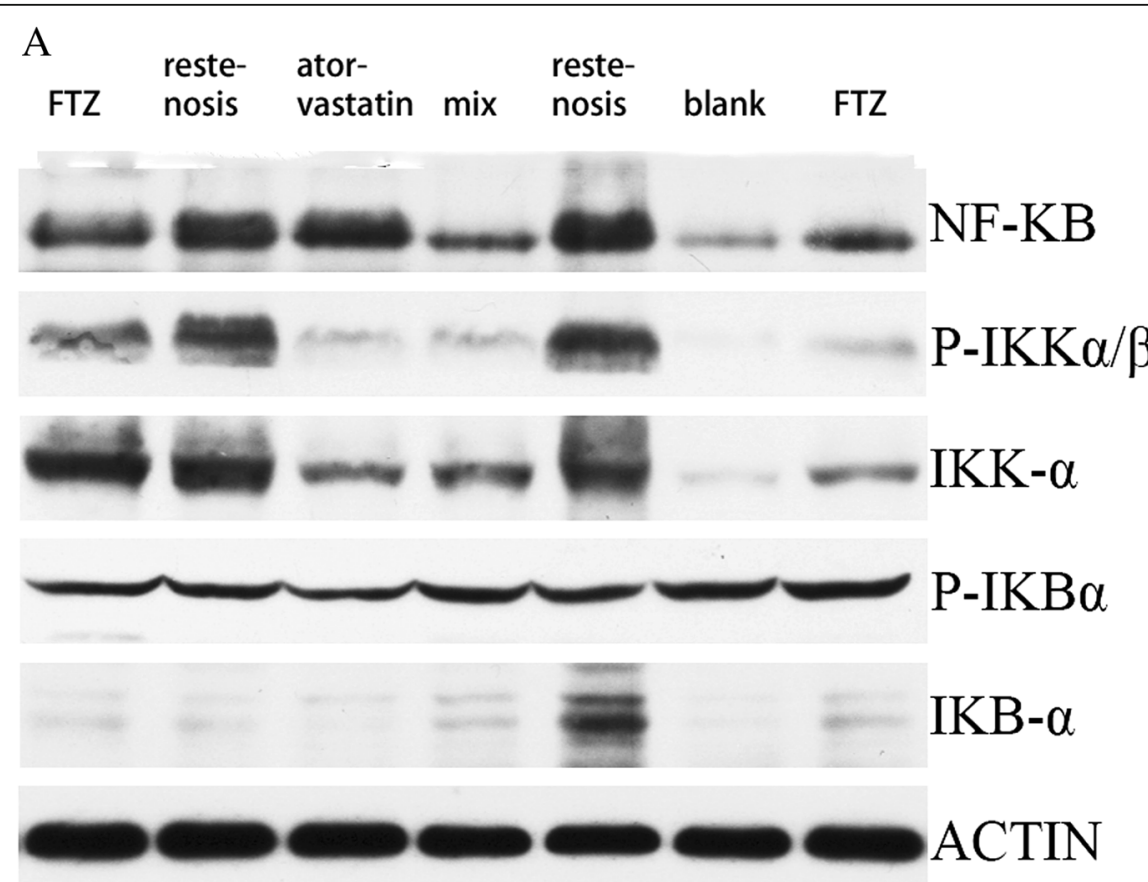

B

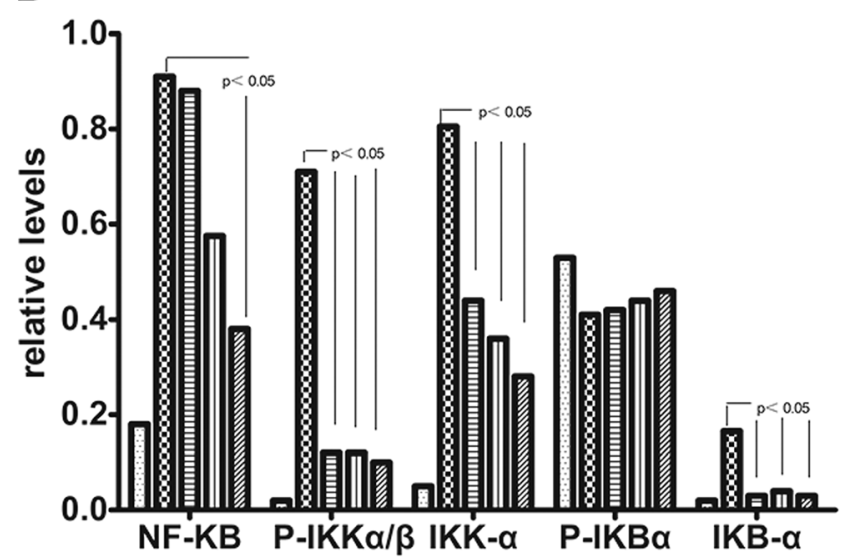

blank group

restenosis group

目 atorvastatin group

四 FTZ group

mix group

Fig. $\mathbf{5}$ The status of NF-KB pathway activation in abdominal aortas of each group. (a) Representative blots showing the levels of NF-KB pathway components in in abdominal aortas of each group. (b) Densitometry analysis of the levels of NF-KB pathway components $(n=3)$. ${ }^{* *} P<0.01$ vs. restenosis group 
could induce inflammation, leading to the formation of restenosis [12]. Lipid-lowering therapy, including statins, was recommended in current PCI guideline. In this study, all rabbits treated with FTZ had significantly lower levels of TC, TG, LDL-C, and VLDL-C. Furthermore, administration of FTZ significantly decreased the restenosis. These results suggest that FTZ is an effective lipid-lowering agent that helps prevent hyperlipidemia-induced restenosis.

Notably, FTZ reduced serum levels of inflammatory factors such as IL-1, IL-6, IL-8, IL-12, CRP, TNF-a and MCP-1. It is known that infiltration of inflammatory cells occurs shortly after endothelial denudation and monocyte infiltration contributes to restenosis [13]. In addition, IL-1 is produced by activated macrophages within atheromatous plaques, and causes pro-inflammatory process involving SMCs proliferation and recruitment of additional inflammatory cells into the plaque and endothelial walls [14]. ICAM-1 is crucial to endothelial leucocytes interaction, and leucocyte migration at the inflammation site. TNF-a plays an important role in vascular inflammation and increases the expression of adhesion molecules [15]. IL-1 is synthesized in endothelial and adventitial cells after balloon injury, and IL-1 related inflammation may play a role in neointima formation $[16,17]$. IL-6 promotes the homing of leukocytes into atherosclerotic plaques [18]. High levels of IL-8 were found in atherosclerotic plaques rich in macrophages [19]. Collectively, we postulate that the beneficial effects of FTZ in preventing restenosis might be mediated by inhibiting inflammatory responses induced by hyperlipidemia.

NF- $\mathrm{KB}$ plays a crucial role in the coordinated transactivation of cytokines and adhesion molecules, and aberrant activation of NF-KB is implicated in a wide range of human diseases, including inflammation, atherosclerosis and vascular diseases [20]. The inhibiting of NF- $\mathrm{kB}$ pathway could reduce neointima formation following vascular injury [21]. Activation of NF- $\mathrm{KB}$ can promote the proliferation of vascular smooth muscle cells by regulating the expression of VCAM-1, MCP-1 and TNF- $\alpha$ [22]. Expression of NF- $\kappa B$ was detected in the nuclei within human atherosclerotic lesions [23]. Our results showed that FTZ significantly reduced NF- $k B$ activation, and inhibited the levels of downstream factors of NF- $\kappa B$, such as IL-1, IL-6, TNF- $\alpha$ and MCP-1, indicating that FTZ targets NF- $\mathrm{kB}$ pathway to ameliorate inflammatory cascade in the vessels.

In this study, FTZ showed similar beneficial effects to atorvastatin on restenosis. Previous studies have found that atorvastatin could reduce the inflammation within the atherosclerotic lesion through the inhibition of NF- $\kappa B$ activity and chemokine expression [24].
The effects of atorvastatin on atherosclerosis may be achieved by the inhibition of the expression of TLR4 and NF-kB p65 [25]. Our experimental data showed similar outcomes of FTZ and atorvastatin treatments. In addition, a combination of atorvastatin and FTZ showed better results than treatment with either drug alone.

There are several limitations in our study. First, we used a balloon injury animal model, which is somewhat different from clinical balloon angioplasty procedures performed in patients. Second, the rabbit responses were solely based upon their reactions to injuries whereas mixed pathological inflammation processes and oxidative stress interactions were shown in human responses to vasculature system under an atherosclerotic milieu. Therefore, further clinical research should be performed to evaluate the effects of FTZ on cardiovascular diseases.

\section{Conclusions}

We established a novel vascular restenosis model through percutaneous puncturing of rabbit arteries with balloon injuries and feeding with high fat diet. Based on this model we demonstrated that FTZ significantly improved lipid profiles and downregulated inflammatory factors to inhibit restenosis probably via the inhibition of NF- $\mathrm{B}$ p pathway. FTZ may be used as a medication for the treatment of restenosis in clinical practice.

\section{Abbreviations \\ CRP: C-reactive protein; FTZ: Fufang-Zhenzhu Tiaozhi Capsule; ICAM- 1: Intercellular adhesion molecule-1; IL-1: Interleukin-1; IL-12: Interleukin-12; IL-6: Interleukin-6; IL-8: Interleukin-8; MCP-1: Monocyte chemoattractant protein-1; PCl: Percutaneous coronary intervention; TNF-a: Tumor necrosis factor-alpha}

\section{Acknowledgements}

Not applicable.

\section{Funding}

This study was supported by Science and Technology Department of Guangdong Province public welfare and capacity-building projects (Guangzhou, China) (No: 2017ZC0212).

\section{Availability of data and materials}

All data and material are available upon request.

\section{Authors' contributions}

$\mathrm{RDZ}$ designed the report and completed the manuscript. TDL help raised animals. ZHZ operated on the rabbits and YQZ was responsible for the postoperative care. ZHZ,YHL, YSY revised the manuscript. ZHZ and JG supervised all the work. All authors read and approved the final manuscript.

\section{Ethics approval}

All animal procedures were performed in accordance with the protocols approved by the Committee for Supervision of Animal Experiments of Guangdong Pharmaceutical University (Guangzhou, China).

Consent for publication

Not applicable.

Competing interests

The authors declare that they have no competing interests. 


\section{Publisher's Note}

Springer Nature remains neutral with regard to jurisdictional claims in published maps and institutional affiliations.

\section{Author details}

${ }^{1}$ Department of Cardiology, Boai Hospital of Zhongshan, Zhongshan 528403, China. ${ }^{2}$ Department of Cardiology, The First Affiliated Hospital of Guangdong Pharmaceutical University, Guangzhou 510080, Guangdong, China.

Received: 21 September 2018 Accepted: 20 November 2018

Published online: 30 November 2018

\section{References}

1. Douglas JS, et al. Coronary stent restenosis in patients treated with Cilostazol. Circulation. 2005;112(18):2826-32.

2. Takeuchi M, et al. Relationship between target lesion revascularization after $\mathrm{PCl}$ and serum cholesterol levels, and influence of HMG-CoA reductase inhibitors in this respect. Jpn J Pharmacol Health Care Sci. 2005;31:808-14.

3. Takashi M, et al. Clinical impact of rapid reduction of low-density lipoprotein cholesterol level on long-term outcome of acute myocardial infarction in the statin era: subanalysis of the ALPS-AMI study. PLoS One. 2015;10(6):e0127835.

4. Cui S, Li K, Ang L, Liu J, Cui L, Song X, Lv S, Mahmud E. Plasma Phospholipids and Sphingolipids identify stent restenosis after percutaneous coronary intervention. JACC Cardiovasc Interv. 2017;10(13):1307-16.

5. Parhofer KG. The treatment of disorders of lipid metabolism. Dtsch Arztebl Int. 2016;113(15):261.

6. Jang JY, et al. Favorable effect of optimal lipid-lowering therapy on neointimal tissue characteristics after drug-eluting stent implantation: qualitative optical coherence tomographic analysis. Atherosclerosis. 2015; 242(2):553.

7. Okopień B, Bułdak Ł, Bołdys A. Current and future trends in the lipid lowering therapy. Pharmacological Reports Pr. 2016;68(4):737.

8. Wang $L$, et al. Potential synergistic effects of Chinese herbal prescription FTZ components detected in blood towards hepatic lipid-modulating targets. Complement Ther Med. 2014;22(5):887.

9. Tang FT, et al. Effects of Fufang Zhenzhu Tiaozhi prescription, a Chinese herbal preparation, on atherosclerosis in ApoE-/- mice and related mechanisms. Chin J Integr Med. 2011:1-7.

10. Guo J, et al. A new TCM formula FTZ lowers serum cholesterol by regulating HMG-CoA reductase and CYP7A1 in hyperlipidemic rats. $J$ Ethnopharmacol. 2011;135(2):299-307.

11. Zhong $X$, et al. Analysis of the constituents in rat serum after Oral Administration of Fufang Zhenzhu Tiaozhi Capsule by UPLC-Q-TOF-MS/ MS. Chromatographia. 2012;75(3-4):111-29.

12. Ucar FM. A potential marker of bare metal stent restenosis: monocyte count - to- HDL cholesterol ratio. BMC Cardiovasc Disord. 2016;16(1):186.

13. Kornowski $R$, et al. In-stent restenosis: contributions of inflammatory responses and arterial injury to neointimal hyperplasia. J Am Coll Cardiol. 1998;31(1):224-30.

14. Ridker PM, Lüscher TF. Anti-inflammatory therapies for cardiovascular disease. Eur Heart J. 2014;35(27):1782.

15. Zhang $\mathrm{H}$, Zhang $\mathrm{C}$. Vasoprotection by dietary supplements and exercise: role of TNFa signaling. Exp Diabetes Res. 2012;2012(1687-5214):972679.

16. Abdellaoui A, Al-Khaffaf H. C-reactive protein (CRP) as a marker in peripheral vascular disease. Eur J Vasc Endovasc Surg. 2007;34(1):18-22.

17. Morton AC, et al. Interleukin-1 receptor antagonist alters the response to vessel wall injury in a porcine coronary artery model. Cardiovasc Res. 2005; 68(3):493.

18. Tzoulaki I, et al. Inflammatory, haemostatic, and rheological markers for incident peripheral arterial disease:Edinburgh artery study. Eur Heart J. 2007; 28(3):354-62.

19. Lindholt JS, Shi GP. Chronic inflammation, immune response, and infection in abdominal aortic aneurysms. Eur J Vasc Endovasc Surg. 2006;31(5):453-63.

20. Yu XH, Zheng XL, Tang CK. Nuclear Factor-KB Activation as a pathological mechanism of lipid metabolism and atherosclerosis. Adv Clin Chem. 2015;70:1.

21. Long $\mathrm{G}$, et al. Sappan lignum extract inhibits restenosis in the injured artery through the deactivation of nuclear factor-KB. Aims Bioengineering. 2014; $1(1): 25-39$.

22. Pamukcu B, Lip GYH, Shantsila E. The nuclear factor - kappa B pathway in atherosclerosis: a potential therapeutic target for atherothrombotic vascular disease. Thromb Res. 2011;128(2):117-23.
23. Kalinina N, et al. Increased expression of the DNA-binding cytokine HMGB1 in human atherosclerotic lesions: role of activated macrophages and cytokines. Arterioscler Thromb Vasc Biol. 2004;24(12):2320.

24. Ortego M, et al. Atorvastatin reduces NF-kappaB activation and chemokine expression in vascular smooth muscle cells and mononuclear cells. Atherosclerosis. 1999;147(2):253-61.

25. Shen DZ, et al. Effect of atorvastatin on expression of TLR4 and NF-kB p65 in atherosclerotic rabbits. Asian Pac J Trop Med. 2013;6(6):493-6.

\section{Ready to submit your research? Choose BMC and benefit from:}

- fast, convenient online submission

- thorough peer review by experienced researchers in your field

- rapid publication on acceptance

- support for research data, including large and complex data types

- gold Open Access which fosters wider collaboration and increased citations

- maximum visibility for your research: over $100 \mathrm{M}$ website views per year

At BMC, research is always in progress.

Learn more biomedcentral.com/submissions 